\title{
Intervalo de Partos em Rebanho Nelore na Amazônia Oriental ${ }^{1}$
}

\section{Francisco Aloísio Cavalcante ${ }^{2}$, Raimundo Martins Filho ${ }^{3}$, Claudio Cabral Campello 4 , Raimundo Nonato Braga Lobo ${ }^{5}$, Gabrimar Araujo Martins ${ }^{6}$}

\begin{abstract}
RESUMO - O estudo foi conduzido para avaliar intervalo de partos (IDP) na raça Nelore, utilizando 578 dados de intervalo de partos de animais criados em regime de pasto, no período de 1988 a 1996. Para as análises estatísticas dos dados, foi usado o Programa SAS (Statistical Analysis System). Foram incluídos no modelo o efeito aleatório do pai da vaca, os efeitos fixos de grupo genético da cria anterior, de ano do parto anterior e do mês do parto anterior e as interações entre grupo genético anterior e mês do parto anterior, grupo genético anterior e ano do parto anterior, além da idade da vaca ao parto como covariável. O IDP foi influenciado pelo pai da vaca e pelo ano do parto anterior. A média estimada por quadrado mínimo e seu respectivo erro-padrão (EP) e coeficiente de variação (CV) foram $431,83 \pm 142,76$ dias e $33,06 \%$, respectivamente. O intervalo de partos, além do efeito de pai, sofreu influência de ambiente. O desempenho reprodutivo do rebanho estudado, para as condições de clima tropical, pode ser considerado como satisfatório, estando de acordo com os parâmetros obtidos por outros autores em diferentes regiões.
\end{abstract}

Palavras-chave: bovinos, intervalo de partos, nelore, reprodução

\section{Calving of Interval on Cattle of Nellore in the Eastern Amazonia}

\begin{abstract}
A study was conducted to evaluate calving interval (CI) in the Nellore breed, using a total of 578 data of calving interval from animals raised in pasture, from 1988 to 1996 . The GLM procedure of the SAS Program was used to analyze the data. The model included the sire of the cow random effect, the fixed effects of genetic group of last calf, of the year and the month of the last calving and the interactions between last genetic group and month of the last calving, genetic group and year of the last calving, and the age of the cow at calving as covariate. The sire of the cow and last year of calving influenced the IDP. The estimated minimum square mean and its respective error standard (EP) and coefficient of variation $(\mathrm{CV})$ were $431.83 \pm 142.76$ days and $33.06 \%$, respectively. The environment in addition of the sire of the cow effect, influenced the calving interval. The reproductive performance of the studied herd, for the tropical climate conditions, could be considered as satisfactory, and is in accordance with the parameters obtained by other authors in different regions.
\end{abstract}

Key Words: bovines, calving interval, Nellore, reproduction

\section{Introdução}

O Brasil destaca-se como um dos cinco países de maior efetivo bovino no mundo, com um rebanho estimado pelo ANUALPEC (1996) em 155 milhões de cabeças, do qual fazem parte 60 milhões de fêmeas aptas à reprodução, que produzem anualmente 33 milhões de bezerros, correspondendo a um índice de natalidade de $55 \%$.

Aliado ao baixo índice de parição, o qual afeta a taxa de desfrute e impossibilita o melhoramento genético, por meio da seleção, em virtude da indisponibilidade de fêmeas para reposição, outros fatores contribuem para reduzir a produtividade dos rebanhos na maioria das regiões do país: deficiências de pastagens e aplicação de técnicas reprodutivas inadequadas. Os baixos índices reprodutivos das raças zebuínas, constantemente mencionados pela literatura científica, impõem prejuízos econômicos graves à atividade e limitam a implementação de programas de melhoramento genético.

O intervalo de partos (IDP) é uma importante característica para avaliação da eficiência reprodutiva e produtiva de um rebanho. Reprodutivamente, ele é constituído pelos períodos de serviço e de gestação e, produtivamente, pelos períodos de amamentação e seco. No aspecto produtivo, o mesmo está direcionado para a matriz gerar pelo menos uma cria por ano e desmando-a, preferencialmente com $50 \%$ do seu peso vivo.

\footnotetext{
${ }^{1}$ Parte da Dissertação de Mestrado em Zootecnia, apresentada à Universidade Federal do Ceará - UFC, pelo primeiro autor.

2 Pesquisador II. Embrapa Acre, Brasil. E.mail: aloisio@cpafac.embrapa.br

3 Professor Titular do Departamento de Zootecnia do- CCA- UFC - Fortaleza - Ceará, Brasil. E.mail: martins@ufc.br

${ }^{4}$ Professor Bolsista - FUNCAP/UECE. Fortaleza - Ceará, Brasil.

${ }^{5}$ Bolsista do CNPq - Departamento de Zootecnia - UFC. Fortaleza - Ceará, Brasil. E.mail: nordeste@ufc.br

${ }^{6}$ Bolsista do CNPq - Departamento de Zootecnia - UFC. Fortaleza - Ceará, Brasil.
} 
A média do intervalo de partos do rebanho brasileiro relatada por vários pesquisadores apresenta grande variação, pois inúmeros fatores, genéticos e de ambiente, influenciam o desempenho da característica.

AROEIRA e ROSA (1982), trabalhando com dados de intervalo de partos, por um período de 18 anos (1955-1972), em rebanho nelore, no município de Terenos-MS, encontraram a média de 514 dias (17,05 meses) e chegaram a conclusão de que a característica foi influenciada $(\mathrm{P} \leq 0,01)$ pelo ano de nascimento e pela idade da vaca.

VIANA e FERREIRA (1982) analisaram 6593 observações de intervalo de partos em rebanho Nelore da Fazenda Santa Maria, município de Acreúna, Estado de Goiás, no período de 1968 a 1978, e obtiveram a média de 13,7 meses para o intervalo de parto. Os autores analisaram os efeitos de grau de sangue, sexo da cria, mês do parto anterior, ano do parto anterior e ordem de parição. Destas variáveis apenas as três últimas foram significativas $(\mathrm{P}<0,05)$

Trabalhando com outro rebanho da mesma raça, no município de Santana do Araguaia, no Estado do Pará, no qual se estudaram os efeito de sexo da cria, mês do parto anterior, ano do parto anterior e ordem de parição, VIANA e FERREIRA (1983) analisaram 1255 dados de intervalo de partos e obtiveram a média de 13,65 \pm 3,7 meses, encontrando a mesma significância $(\mathrm{P}<0,05)$ verificada no rebanho analisado anteriormente.

Dois rebanhos da Companhia de Desenvolvimento do Vale de São Francisco (CODEVASF) foram pesquisados por MIRANDA et al. (1982), nos municípios de Formoso-BA e Brasilândia-MG, com 872 e 1768 observações, respectivamente. Os autores encontraram média de 468 dias (15,53 meses) e 441 dias (14,63 meses) de intervalo de partos, para os rebanhos de Formoso e Brasilândia. As análises mostraram que o ano de nascimento, mês de nascimento e ordem do parto estavam significativamente associados à característica, sendo que, no rebanho de Formoso, o mês não foi incluído na análise.

OLIVEIRA FILHO et al. (1986a) analisaram 748 intervalos de partos originados de inseminações artificiais de um rebanho em Lençóis Paulista-SP, no período de 1977 a 1984 , e obtiveram média de 536,82 $\pm 5,60$ dias (17,81 meses). O ano e a ordem de parição exerceram efeito significativo $(\mathrm{P}<0,01)$, ao contrário dos efeitos do mês do parto, do sexo do produto, do tipo de cobertura (monta natural x I.A) e do inseminado, que não influenciaram esta característica. Em trabalhorealizado na mesmaépoca, no município de
Gavião Peixoto-SP, OLIVEIRA FILHO et al. (1986b) estimaram o intervalo médio de parto para 275 observações, obtendo o valor de 398,73 $\pm 1,75(13,22$ meses) dias, tendo sido significativos os efeitos de grupo genético, ano do parto e ordem de parição, ao contrário da estação em que ocorreu o parto e do sexo do produto.

Trabalhando na comparação entre rebanhos da raça Nelore, CARDELLINO e PONS (1987) estudaram intervalos de partos de dois rebanhos no Estado do Paraná, que foram denominados de A e B. O rebanho A era constituído por 85 vacas, com dados coletados no período de 1976 a 1978, o qual forneceu 165 observações. O rebanho B era constituído por 161 vacas, e os dados do período de 1970 a 1978 originaram 472 observações. Os autores encontraram para as médias do intervalo de partos valores de $477 \pm 43$ dias (15,83 meses) e $533 \pm$ 7,64 dias (17,68 meses) para os rebanhos A e B, respectivamente. Foram significativas a interação ano $\mathrm{x}$ estação de nascimento, para as análise do rebanho A, e vaca, ano, interação sexo $\mathrm{x}$ ano de nascimento e idade da vaca ao parto, para o rebanho B.

MARTINS FILHO et al. (1991) analisaram informações de 318 fêmeas da Agropecuária CFM, criadas no Estado de São Paulo, no período de 1984 a 1989, encontrando média de 394,42 dias (13,09 \pm 0,95 meses) para o intervalo de partos. O manejo das propriedades foi fundamental para a boa média de intervalo de parto obtida, pois os autores constataram que os valores encontrados foram inferiores à maioria dos dados já publicados para as raças zebuínas.

Em condição de pastagens artificiais formadas por Panicum maximum Jacq (Colonião) e por variedades de Brachiaria, MARTINS FILHO et al. (1994) encontraram média de 372,2 dias (12,46 meses) para os intervalos de partos, trabalhando com rebanho nelore de fêmeas filhas de 29 touros, no município de Araçatuba-SP, média abaixo da encontrada por SOUZA et al. (1994), também, em rebanhos nelore, no mesmo estado. Na análise de variância do trabalho de MARTINS FILHO et al. (1994), foram incluídos os efeitos do pai da vaca, ano e mês de nascimento, sexo e ordem de parição; os resultados revelaram significância $(\mathrm{P}<0,01)$ para o mês de nascimento e a ordem de parição, sendo a média do intervalo de parto inferior àquelas constantes na literatura, para a raça, o que foi atribuído, em parte, às condições de manejo do rebanho.

O objetivo deste estudo foi verificar os efeitos genéticos e de ambiente que interferem na caracte- 
rística intervalo de partos, em um programa de inseminação artificial em rebanho da raça Nelore, puro de origem (PO), no período de 1988 a 1996.

\section{Material e Métodos}

Foram analisadas 587 observações de intervalo de partos de 405 matrizes nelores criadas a pasto, na Fazenda Entre Rios, no município de Godofredo Viana, localizada no Estado do Maranhão, no período de 1988 e 1996.

As matrizes nelores foram sincronizadas e inseminadas com doses de sêmen de touros das raças Marchigiana ou Nelore, no período de julho a dezembro. Após dois meses de inseminadas, foram apalpadas.

Os dados foram tirados dos formulários de nascimento, inseminação artificial dos arquivos do Escritório Técnico Regional da ABCZ, em Fortaleza-CE e de fichas zootécnicas da propriedade. O intervalo de partos de cada matriz foi calculado subtraindo-se a data de duas ou mais parições.

Os dados foram analisados pelo procedimento GLM do Programa SAS (Statistical Analysis System, 1990), por intermédio do seguinte modelo: efeitos fixos de grupo genético da cria anterior, mês e ano do parto anterior, ano e mês do parto, interações grupo genético $\mathrm{x}$ mês do parto anterior e grupo genético $\mathrm{x}$ ano do parto anterior, enquanto o efeito do pai da vaca foi considerado aleatório e a idade da vaca ao parto, usada como covariável.

$$
\begin{gathered}
Y_{i j k l m n}=\mu+a_{i}+G_{J}+M_{K}+A_{1}+G^{x} M_{j K}+ \\
G^{x} A_{j 1}+b_{1}\left(I_{i k l m}-\bar{I}\right)+e_{i j k l m n}
\end{gathered}
$$

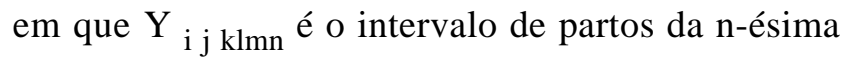
vaca, filha do pai $\mathrm{i}$, com cria do grupo genético ${ }_{\mathrm{j}}$, parida no mês ${ }_{\mathrm{K}}$, do ano $_{1}$, com idade ${ }_{\mathrm{m}}$, por ocasião do parto; $\mu$, a média geral para a característica estudada; $a_{i}, o$ efeito aleatório do pai da vaca; $G_{\mathrm{J}}$, o efeito fixo do grupo genético da cria anterior; $G_{1}$, Nelore; $G_{2}$, Marchigiana; $M_{K}$, o efeito fixo do mês do parto anterior; $A_{1}$, o efeito fixo do ano do parto anterior; $\mathrm{G} \times \mathrm{M}_{\mathrm{jK}}$, o efeito da interação entre grupo genético e mês do parto anterior; $\mathrm{G} \mathrm{x} \mathrm{A} \mathrm{j}_{\mathrm{j}}$, o efeito da interação entre grupo genético e ano do parto anterior; $b_{1}$, o coeficiente de regressão do termo linear, da idade da vaca ao parto, sobre a característica estudada; $\mathrm{I}_{\mathrm{ijklmn}}$, a idade da $n \stackrel{\text { ésima }}{ }$ vaca, filho do pai $i$, com cria do grupo genético j, parida no mês k, do ano I, com idade m, por ocasião ao parto; $\overline{\mathrm{I}}$, as médias das idades das vacas ao parto; (I - I), o efeito fixo, linear da idade da vaca ao parto; e $e_{i j k l m n}$, o erro aleatório, normal, independentemente distribuído, com média zero e variância $\sigma^{2}$.

\section{Resultados e Discussão}

A média ajustada e o coeficiente de variação foram, respectivamente, $431,83 \pm 142,76$ dias $(14,20$ meses) a $33,06 \%$. A média obtida foi superior aos valores (13,65 meses, meses, 13,14 meses e 13,58 meses) de VIANA e FERREIRA (1983), trabalhando em rebanho no Estado Pará, de MARTINS FILHO et al. (1991) e SOUZA et al. (1994), na Amazônia Oriental e em outras regiões, respectivamente.

Valor superior (17,39 meses) em região diferente foi encontrado por PEROTTO et al. (1994). Valor inferior (12,46 meses), também em ambientes diferentes, foi obtido MARTINS FILHO et al. (1994). Conforme Tabela 1, foi verificado efeito significativo $(\mathrm{P}<0,05)$ do pai da vaca e do ano do parto anterior sobre a característica de IDP. Observou-se, para a característica IDP, que houve efeito significativo do pai da vaca $(\mathrm{P}<0,05)$, confirmando os resultados de DIAS e OLIVEIRA (1994) e CAMPELLO (1996). No entanto, MARTINS FILHO et al. $(1991,1994)$ não observaram significância para este efeito. Possivelmente esta discordância entre os autores tenha relação com aspectos genéticos dos reprodutores utilizados em seus trabalhos ou reflexo na eficácia funcional do sistema endócrino e do metabolismo. Isto provavelmente levaria a algumas progênies destes touros a apresentarem intervalos menores em parições subseqüentes.

Provavelmente, o efeito significativo do pai sobre o IDP tenha origem no fato de que, nas condições em que este trabalho foi desenvolvido, o período de serviço foi significativamente influenciado pelo mesmo efeito. Conforme foi justificado, a precocidade relativa de involução uterina e retorno à atividade ovariana pós-parto, entre filhas de touros diferentes comparadas entre si, seria reflexo da variação na eficiência funcional do sistema endócrino e do metabolismo com um todo, relacionada a aspectos genéticos. Este melhor funcionamento levaria as filhas de alguns touros a apresentar menores intervalos de parições sucessivas, por meio de reduções nos períodos de serviço. $O$ efeito do grupo genético da cria anterior (Nelore ou Marchigiana) nãofoi significativo $(\mathrm{P}>0,05)$ para IDP. As médias de IDP foram 434,51 $\pm 21,93$ e 414,79 $\pm 18,92$ para Nelore e Marchigiana, respectivamente.

Foi verificado efeito significativo do ano do parto anterior $(\mathrm{P}<0,05)$ sobre a variação do IDP. Os valores para a característica apresentaram crescimento acentuado de 1988 a 1990, seguido de redução paulatina entre 1990 e 1994. A partir de 1994, houve evidente 
Tabela 1 - Análise de variância para o intervalo de parto (IDP) Table 1 - Analyses of variance for calving interval (Cl)

Fonte de variação $\quad$ Graus de Quadrado F

Source of variation liberdade médio

\begin{tabular}{lccc} 
& $\begin{array}{c}\text { Degrees of } \\
\text { freedom }\end{array}$ & $\begin{array}{c}\text { Mean } \\
\text { square }\end{array}$ & \\
\hline Pai da vaca & 38 & 31332,33 & $1,54^{*}$
\end{tabular}

Sire of cow

Grupo genético anterior(GGA) $1 \quad 19647,34 \quad 0,96$

Last genetic group

Ano do parto anterior (APA) $7 \quad 41604,89 \quad 2,04^{*}$

Last year calving

Mês do parto anterior (MPA) $11 \quad 23332,81 \quad 1,14$

Last month partum

$\begin{array}{llll}\text { Interação GGA x MPA } & 11 & 21190,74 & 1,04\end{array}$

Interaction $L G G \times A C M$

Interação GGA ${ }^{\mathrm{x}} \mathrm{APA}$

Interaction $L G G \times Y C L$

Idade da vaca ao parto

Age cow calving

$\begin{array}{lll}\text { Resíduo } & 510 \quad 20382,39\end{array}$

Error

${ }^{*} \mathrm{P}<0,05$.

declínio nos valores de IDP (Tabela 2 e Figura 1). VIANA e FERREIRA (1982), CARDELLINO e PONS (1987) e GONÇALVES et al. (1996), em trabalho anterior, confirmaram esta significância. Entretanto, ALENCAR e BUGNER (1989), MARTINS FILHO et al. (1991) e CAMPELLO (1996) não encontraram significância para o efeito, na característica.

A discordância entre os autores pode ser explicada pelas condições ambientais e de manejo alimentar, pois, uma vez que os trabalhos de VIANA e FERREIRA (1982) e CAMPELLO (1996) se desenvolveram em condições diferentes, pode-se justificar esta discordância, já que o primeiro ocorreu em uma época de implantação da propriedade, com a formação de pastagens e compra de rebanho nelore comercial, ainda se fazendo controle sanitário e sem suplementação na época de estiagem, entre outros, e o segundo constituído por um rebanho geneticamente bem superior, pastagens já formadas, bem manejadas, com controle sanitário eficiente e suplementação com volumoso e concentrado na época de estiagem. Essa diferença acentuada de condições, provavelmente, provocou esta discordância nos resultados.

Os efeitos significativos do ano do parto anterior podem ser entendidos como decorrentes das mudanças de ambiente, caracterizando um parâmetro essencialmente influenciado pelo meio. O efeito de ambiente deve ser entendido como todo efeito que não seja de origem genética, incluindo o manejo concernente à amamentação indiscriminada, a qual exerce efeito, por vezes adverso, sobre o eixo hipotalâmicohipofisário-gonadal.

O comportamento do IDP também reflete a tendência observada no período de serviço e, provavelmente, se deve às alterações administrativas e de manejo pelas quais a propriedade passou ao longo do período considerado.

As mudanças anuais de inseminadores, as práticas de recuperação de pastagens que se haviam degra-

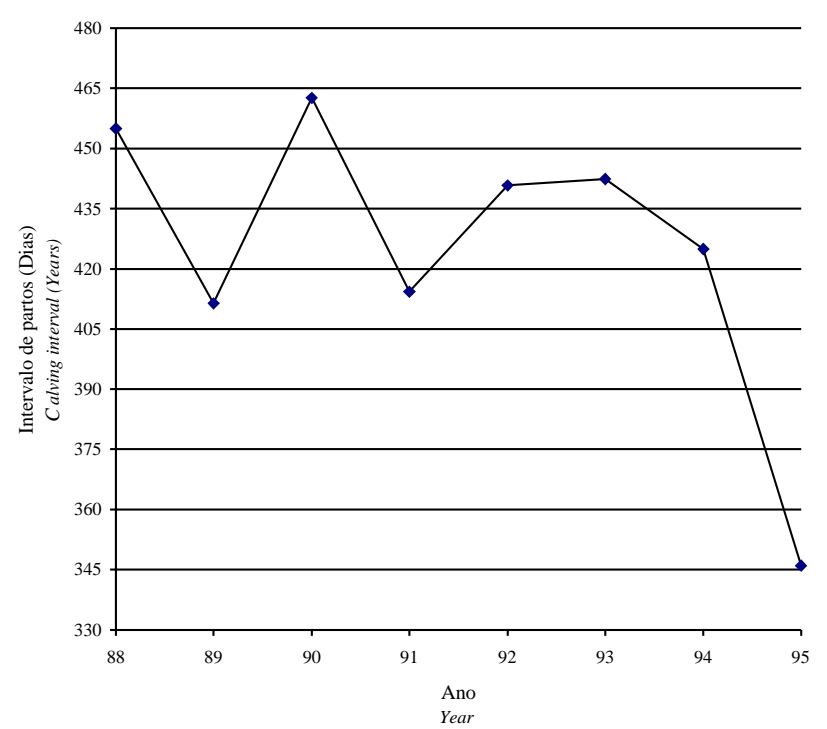

Figura 1 - Médias ajustadas para o intervalo de parto (IDP), de acordo com efeito do ano do parto anterior (APA).

Figure 1 - Adjusted means for calving interval (Cl), according to the effect on last year calving ( $L Y C)$.

Tabela 2 - Médias ajustadas para o intervalo de parto (IDP) de acordo com o efeito do ano do parto anterior (APA)

Table 2 - Adjusted means for calving interval, according (Cl) to the effect on last year calving ( $L Y C)$

\begin{tabular}{|c|c|c|}
\hline $\begin{array}{l}\text { Ano do parto } \\
\text { anterior }\end{array}$ & $\begin{array}{l}\text { Número de } \\
\text { observações }\end{array}$ & $\begin{array}{l}\text { Médias ajustadas } \\
\text { (Dias) }\end{array}$ \\
\hline $\begin{array}{l}\text { Last year } \\
\text { calving }\end{array}$ & $\begin{array}{l}\text { Number of } \\
\text { observations }\end{array}$ & $\begin{array}{c}\text { Adjusted means } \\
\text { (Days) }\end{array}$ \\
\hline 1988 & 34 & $454,94 \pm 36,21$ \\
\hline 1989 & 54 & $411,36 \pm 26,63$ \\
\hline 1990 & 91 & $462,58 \pm 22,47$ \\
\hline 1991 & 98 & $414,24 \pm 22,14$ \\
\hline 1992 & 131 & $440,81 \pm 28,40$ \\
\hline 1993 & 90 & $442,32 \pm 22,96$ \\
\hline 1994 & 68 & $424,97 \pm 26,23$ \\
\hline 1995 & 21 & $345,99 \pm 35,71$ \\
\hline
\end{tabular}


dado ao longo do tempo, a decisão de se iniciar um programa de exames ginecológicos e sincronizações de cio e os processos de seleção de matrizes (descartes das portadoras de problemas reprodutivos) são exemplos de fatores que possivelmente tenham interferido sobre a variação do IDP no intervalo considerado.

Embora haja possibilidade de influência ambiental, como variações de pluviosidade entre os anos estudados, com repercussão sobre a oferta de alimentos para o rebanho, a inexistência de informações a este respeito limita a possibilidade de se fazer um paralelo entre esta possível causa e seus efeitos.

\section{Conclusões}

O intervalo de partos, além do efeito de pai, sofreu influência de ambiente.

O desempenho reprodutivo do rebanho estudado, para as condições de clima tropical, pode ser considerado como satisfatório, estando dentro dos parâmetros obtidos por outros autores.

\section{Agradecimento}

Ao grupo Edson Queiroz, pela cessão dos dados para a realização deste trabalho.

Aos revisores da Revista Brasileira de Zootecnia, pelo exame detalhado, pelas sugestões valiosas e sucessivas correções.

\section{Referências Bibliográficas}

ALENCAR, M.M., BUGNER, M. 1989. Desempenho produtivo de fêmeas das raças Canchim e Nelore. Segundo parto. Pesq. Agropec. Bras., 24(10):1217-1220.

ANUALPEC. 1996. Anuário Estatístico da Pecuária de Corte. FNP Consultoria \& Comércio, São Paulo. 312p.

AROEIRA, J.A.D.C., ROSA, A.N. 1982. Desempenho reprodutivo de um rebanho Nelore. Pesq. Agropec. Bras., 17(2):337-343.

CAMPELLO, C.C. Fatores genéticos e de ambiente que influenciam características reprodutivas em fêmeas da raça nelore. Fortaleza: UFC, 1996. 62p. Dissertação (Mestrado em Zootecnia) - Universidade Federal do Ceará, 1996.

CARDELLINO, R.A., PONS, S.B. 1987. Parâmetros genéticos do intervalo entre partos em bovinos da raça Nelore. Pesq. Agropec. Bras., 22(3):305-310.

DIAS, F.M.G.N , OLIVEIRA, H.N. Efeito da consangüinidade sobre o intervalo entre partos (IEP) de fêmeas da raça nelore. In: REUNIÃO ANUAL DA SOCIEDADE BRASILEIRA DE ZOOTECNIA, 31, 1994. Maringá. Anais... Maringá: SBZ, 1994, p.173.
GONÇALVES, J.N.S., SCARPATI, M.T.V., NARDON,R.F. Avaliação da fertilidade real e da capacidade mais provável de fertilidade real de matrizes de um rebanho da raça nelore. In: REUNIÃO ANUAL DA SOCIEDADE BRASILEIRA DE ZOOTECNIA, 33, 1996. Fortaleza. Anais... Fortaleza: SBZ, 1996, p.368.

MARTINS FILHO, R., LÔBO, R.B., SILVA, P.R. Efeitos genéticos e de meio sobre características reprodutivas de fêmeas da raça Nelore. In: REUNIÃO ANUAL DA SOCIEDADE BRASILEIRA DE ZOOTECNIA, 31, João Pessoa, 1994. Anais... João Pessoa: SBZ, 1991, p.572.

MARTINS FILHO, R., LÔBO, R.B., OLIVEIRA, S.M.P. Idade ao primeiro parto e intervalo entre partos em rebanho bovino da raça Nelore. In: REUNIÃO ANUAL DA SOCIEDADE BRASILEIRA DE ZOOTECNIA, 31, Maringá, 1994. Anais... Maringá: SBZ, 1994, p.175.

MIRANDA, J.J.F., CARNEIRO, G.G., FONSECA, C.G. 1982. Fatores ambientais e genéticos relacionados com o intervalo entre partos na raça nelore. Arq. Esc Vet. UFMG, 34(2):381-387.

OLIVEIRA FILHO, E.B., DUARTE, F.A.M., GONÇALVES, A.A.M. Eficiência reprodutiva em um rebanho Nelore: período de serviço e intervalo entre partos. In: REUNIÃO ANUAL DA SOCIEDADE BRASILEIRA DE ZOOTECNIA, 23, 1986a. Anais... Campo Grande: SBZ, 1986a. p.362.

OLIVEIRA FILHO, E.B., DUARTE, F.A.M., LOBO, R.B. Aspectos da eficiência reprodutiva de um rebanho Nelore: efeitos genéticos e de meio ambiente. In: CONGRESSO BRASILEIRO DE MEDICINA VETERINÁRIA, 20, Cuiabá, 1986. Anais... Cuiabá: SBMV, 1986b. p.200.

PEROTTO, D., JOSÉ, W.P.K., ABRAHÃO, J.J.S. Idade ao primeiro parto e intervalo entre partos de fêmeas bovinas Nelore e de mestiças Guzerá x Nelore, Red Angus x Nelore e Marchigiana x Nelore. In: REUNIÃO ANUAL DA SOCIEDADE BRASILEIRA DE ZOOTECNIA. 31, 1994. Maringá. Anais... Maringá: SBZ. 1994. p.176.

Statistical Analysis System - SAS. 1990. User' s guide. SAS Institute Inc. Cary, NC. 142p.

SOUZA, J.C., RAMOS, A.A, FERRAZ FILHO, P.B. Estudo dos intervalos de partos de matrizes da Raça Nelore. In: REUNIÃO ANUAL DA SOCIEDADE BRASILEIRA DE ZOOTECNIA, 31, 1994. Maringá. Anais.. Maringá: SBZ, 1994, p.178.

VIANA, H.U., FERREIRA, P.R.C. 1982. Efeitos de alguns fatores de meio no intervalo entre partos de fêmeas nelore. Pesq. Agropec. Bras., 17(8):1227-1232.

VIANA, H.U., FERREIRA, P.R.C. 1983. Fatores ambientais e efeitos do sexo no intervalo entre partos de fêmeas nelore. Pesq. Agropec. Bras., 18(8):937-942.

Recebido em: 09/02/99

Aceito em: 21/09/99 